\title{
MATHEMATICAL SUPPORT OF CRISIS PLANNING
}

It is impossible to eliminate the occurrence of crises and crises situations in society, nature and in production processes. That is why it is necessary to search for such paths and procedures, which would minimise the crises processes and consequences. Methods of mathematical statistics provide valuable information for crises planning in the sphere of mathematical forecasting.

\section{Introduction}

The existence of life on earth is linked with nature and the living environment, which people try to modify according to their needs. These activities are connected with a number of risks, which endanger not only the environment but also people themselves. Emergency situations and various crises events have accompanied and will accompany our lives forever. They are an inseparable part of our lives and people have to find ways how to prevent them and, in case they occur, how to eliminate their consequences and minimise losses and damages. In case we apply appropriate preparations and technological procedures, it is possible, to a certain extent, affect the occurrence and elimination of crises situations consequences.

People have to base their future on knowledge and understanding of the past. Society tries to predict and appropriately respond to the origin of crises situations using crisis planning and by creation of material, technical, technological and human resources. Identification of consequences and patterns of emergency situations and forecasting of their occurrence and consequences are the main prerequisite for their successful solution. Authorities and people interested in advance try to provide measurements, which make their early and effective control possible.

Crisis planning should offer and prepare measurements for solution of crises situations, which could occur sometime in the future. It follows the experience gained in the past and tries to prepare such plans and measurements that would prevent the origin of emergency situations or that would minimise their consequences. That is why forecasting is a very crucial task of crisis planning.

As people gradually reveal the nature of natural and social phenomena, they also create systems for monitoring of the risk factors involved. This enables to identify dependencies of phenomena such as natural disasters, calamities, catastrophes etc., and to predict their occurrence as well as to provide measurements for losses and damages minimisation. In certain cases this method has enabled to get natural elements under control with no damages. This is one of the objectives of the crisis management strategy.
From the point of view of purposes specified and means used we can define political, diplomatic, economical, safety, military and scientific-technical crisis management strategy.

There exists a universally acceptable principle. The means of prevention and solution of crises and crises events incurred are in due proportion to the knowledge and technical potential of the mankind. That is why elaboration and actual application of mathematical methods to crisis planning tasks is one of the objectives of current science. Contemporary mathematical statistics methods enable not only statistical bulk data processing, but also acquisition of a lot of information of high predicative capacity.

\section{Mathematical Statistics}

Mathematical statistics and statistic analysis methods are more and more used in various subject fields. Statistics is a theoretical branch of knowledge. It studies the state and development of numerically formulated bulk phenomena. These phenomena can occur in various spheres concerning people and their activities, animals, things, organisations, institutions, societies, as well as production, management, natural phenomena and others. Mathematical statistics does not only deal with quantitative part of bulk phenomena, but also with their qualitative part. Using statistical analysis, it can also reveal various regularities, dependencies and development tendencies of the set of elements. These revealed regularities as well as identified coherence in the state and development of phenomena are later used in application from parts to the whole, from partial to universal as well as in forecasting of bulk phenomena future development.

\section{Forecasting by Means of Mathematical Statistics}

Statistical set is a basis for any statistical enquiry. It comprises a sample of objects (elements, phenomena, actions, elements, measurements, etc.). There are two kinds of statistical sets: primary (contains all elements) and selected (contains only a certain subset of a primary set). The set range should be representative and as

\footnotetext{
* I. Milata, L. Rošteková, V. Kašpar, Z. Dvořák

Department of Technical Sciences and Informatics, Faculty of Special Engineering, University of Žilina, Slovakia, E-mail: milata@fsi.utc.sk
} 
wide as possible to compensate incidental, irrelevant and accidental influences.

For statistical forecasting it is necessary to balance the examined set by such a curve that would bind to the elements of the set as much as possible. The function of this curve enables us to perform calculation of set indicators in other spheres than those measured beforehand or to perform mathematical forecasting.

Statistical research is divided into four basic consecutive parts:

1. Statistical detection or material collection. When collecting statistical data it is possible to use these basic methods:

- Reports - information of news units

- Measurements of real actions (monitoring)

- Questionnaires filled in subjects.

2. Material classification - characteristics calculation.

3. Statistical analysis (in this case correlation and regression analysis)

4. Adoption of hypotheses and estimations, their testing.

Correlation is a statistical relation of two or more variables. It presents information on statistical relation of phenomena, determines the strength of this relation, but it does not inform on their cause. The main result of a correlation is called the correlation coefficient $\mathrm{r}$ or correlation index. Correlation coefficient $r$ varies from 0 to 1 . It indicates how expected (anticipated) values, expressed by perfect correlation line, match real data. A coefficient of 1 gives a perfect fit.

It is possible to use analytical method of correlation to test relationship of two data sets. Positive correlation is a relation in which the high values in one set match high values in the second set. Negative correlation means that low values in one set match high values in the second set. If there is no connection between values in both sets, we say there is zero correlation.

Correlation coefficient squared

$$
r^{2}=1-\frac{\sum_{i=1}^{n}\left(y_{i}-y(x)\right)^{2}}{\sum_{i=1}^{n}\left(y_{i}-\left\langle y_{i}\right\rangle\right)^{2}}
$$

Definitions $x$ is $x$ - coordinate of a data point $y$ is $y$-- coordinate of a data point $n$ is number of data points

$$
\begin{aligned}
& \left\langle y_{i}\right\rangle=\frac{\sum_{i=1}^{n} y_{i}}{n} \\
& r=0.0-0.3 \\
& r=0.3-0.7 \\
& r=0.7-0.9 \\
& r=0.9-1.0
\end{aligned}
$$

Regression determines the shape of statistical relation.

Regression analysis is, by means of perfect regression line, used for graphic representation of orientation in data and for the study of forecasting problems. By means of regression analysis, through extension of perfect regression line it is possible to determine values before and after displayed data. This way we can perform mathematical forecast. Accuracy of mathematical forecasting bears a proportion to the strength of correlation relation.

Methods of least squares are used to define coefficient of a regression function. This means that we are looking for such a function, where the difference between sums of squares of the offsets of measured and theoretical data is minimal. From the point of geometrical view it means that we are looking for such a curve that would fit the best to individual points. Function of this curve should be as easy as possible, so it can be easily used for calculation of other necessary values. There exist various shapes of regression lines.

Most frequently we use these functions:

- linear

- exponential

- power

- logarithmic

- polynomial

\section{Linear Function Equation}

The easiest equation of measured parameters is their equation by linear function. Linear best-fit line is a line used in simple linear data sets. Data is linear, when the course of data points resembles a line. Linear best-fit line usually graphs that something constantly rises or decreases.

$$
y=a+b x
$$

Calculation of $\mathrm{a}, \mathrm{b}$ constants:

$$
a=\frac{\sum_{i=1}^{n} x_{i}^{2} \sum_{i=1}^{n} y_{i}-\sum_{i=1}^{n} x_{i} \sum_{i=1}^{n} x_{i} y_{i}}{n \sum_{i=1}^{n} x_{i}^{2}-\left(\sum_{i=1}^{n} x_{i}\right)^{2}}
$$

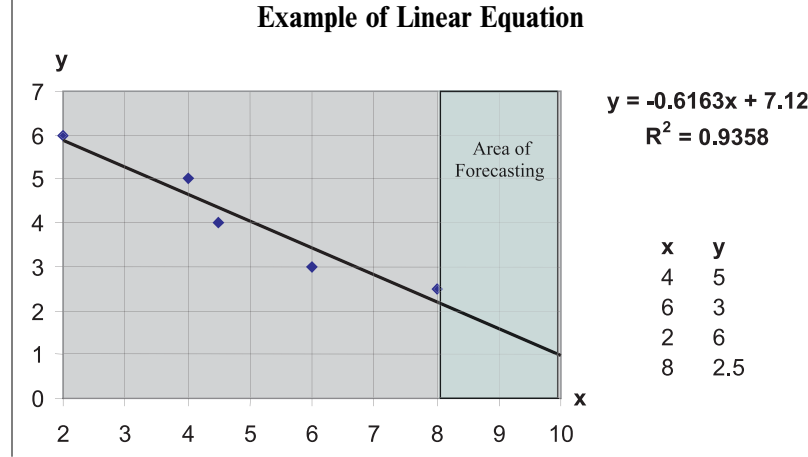




$$
a=\frac{\sum_{i=1}^{n} x_{i} y_{i}-\sum_{i=1}^{n} x_{i} \sum_{i=1}^{n} y_{i}}{n \sum_{i=1}^{n} x_{i}^{2}-\left(\sum_{i=1}^{n} x_{i}\right)^{2}}
$$

\section{Power Function Equation}

Power best-fit line is a curve used in the case of data which compare rising values measured in specific intervals. E.g. car acceleration at intervals of $1 \mathrm{sec}$. It is not possible to graph a power bestfit line when the data includes zero or negative values.

$$
y=a x^{b}
$$

Calculation of $\mathrm{a}, \mathrm{b}$ constants:

$$
\begin{aligned}
\log a & =\frac{n \sum_{i=1}^{n} x_{i} \log y_{i}-\sum_{i=1}^{n} x_{i} \sum_{i=1}^{n} \log y_{i}}{n \sum_{i=1}^{n} x_{i}^{2}-\left(\sum_{i=1}^{n} x_{i}\right)^{2}} \\
\log b & =\frac{n \sum_{i=1}^{n} x_{i}^{2} \sum_{i=1}^{n} \log y_{i}-\sum_{i=1}^{n} x_{i} \sum_{i=1}^{n} x_{i} \log y_{i}}{n \sum_{i=1}^{n} x_{i}^{2}-\left(\sum_{i=1}^{n} x_{i}\right)^{2}}
\end{aligned}
$$

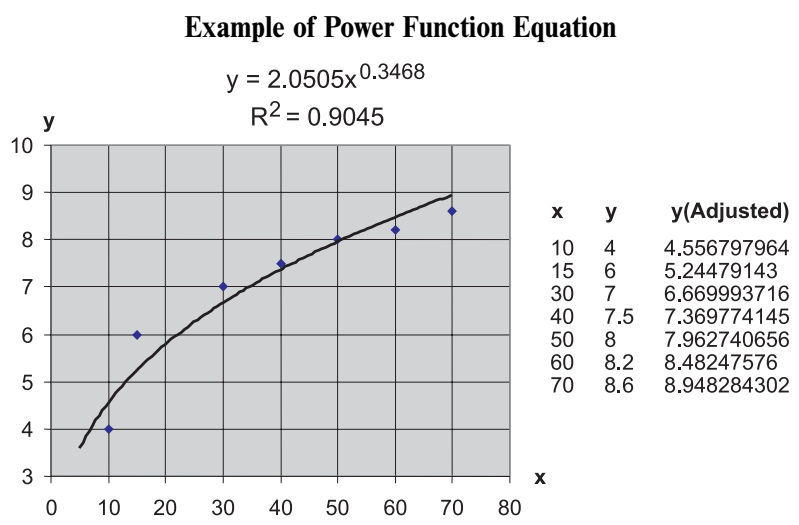

\section{Logarithmic Function Equation}

Logarithmic best-fit line is an adjusted curve used when data is quickly rising or decreasing and then continuously adjusting. It is possible to include positive and negative values when graphing logarithmic best-fit line.

$$
y=a \ln (x)+b
$$

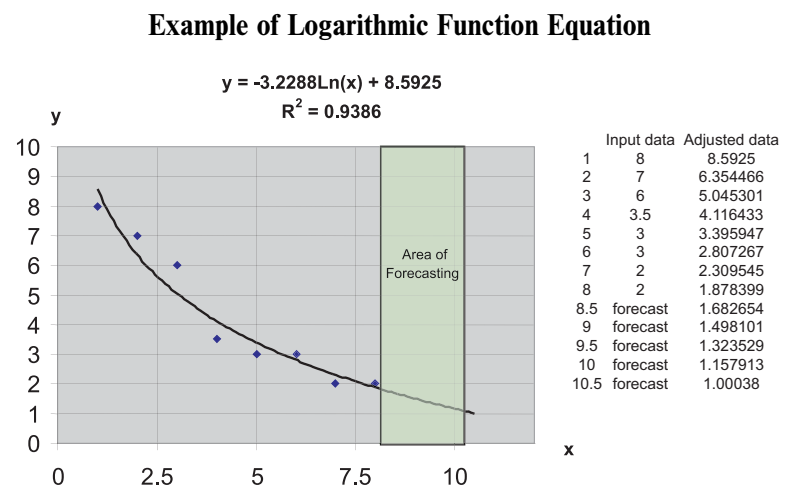

\section{Polynomial Function Equation}

Polynomial best-fit line is a curve used when data varies and it is not possible to approximate it by a simpler function. The degree of polynomial can be defined by a number of data variation or by a number of curvatures (maxima and minima) in a curve. Degree 2 usually has one local maximum. Degree 3 usually has one or two local maxima. Degree 4 usually has as many as three local maxima.

$$
y=a+b_{1} x+b_{2} x^{2}+\ldots+b_{6} x^{6}
$$

\section{Degree 3 Polynomial Function Equation}

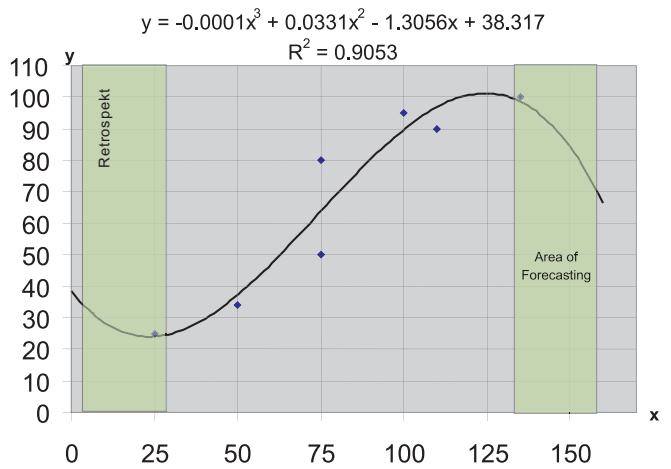

\section{Exponential Function Equation}

Exponential best-fit line is a curve, which is used in case when data values rise or decrease markedly. It is not possible to graph this line, when data includes zero or negative values.

$$
y=a e^{b x}
$$

This is a practical air transport example for exponential function equation.

This graph shows a statistical set containing real observed data. It presents the number of fatal accidents per 1 million kilometres 
Graph of Fatal Accidents per 1 mil. km Flown
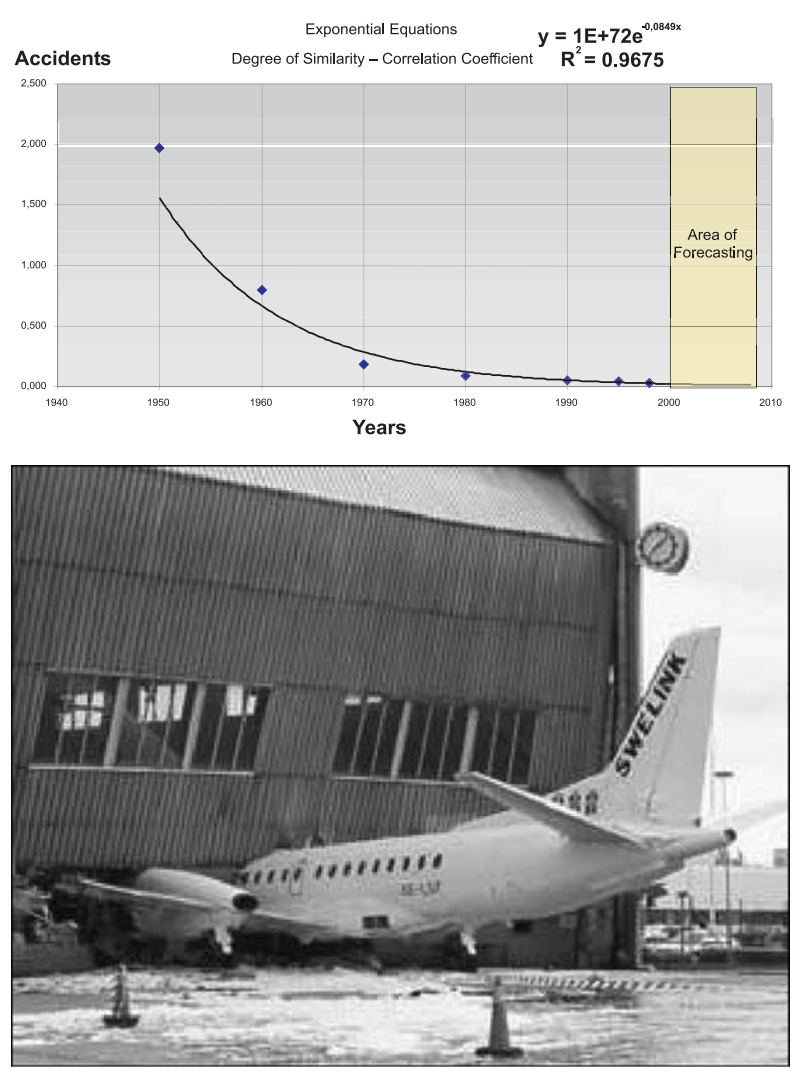

flown in the period of years 1950 - 1998. The set has been equated by exponential function. Correlation coefficient of 0.9682 is quite high, so it is possible to use exponential function for statistic forecasting. This has been realized numerically and graphically for the period of years 2004 - 2008. Larger number of real data indicates more accurate results.

Forecasted data is extremely valuable information necessary for crisis planning strategy. This and similarly evaluated real information can be applied to every airport. This enables to prepare appropriate size of local rescue forces and means, as well as to

\begin{tabular}{|c|c|c|}
\hline Year & Fatal Accidents & Adjusted \\
\hline \multicolumn{3}{|l|}{ Real } \\
\hline 1950 & 19.000 & 15.7535322 \\
\hline 1960 & 8.000 & 6.77178427 \\
\hline 1970 & 1.800 & 2.91090668 \\
\hline 1980 & 0.900 & 1.25127697 \\
\hline 1990 & 0.500 & 0.53787161 \\
\hline 1995 & 0.450 & 0.35264782 \\
\hline 1998 & 0.300 & 0.27374031 \\
\hline \multicolumn{3}{|c|}{ Forecasted } \\
\hline 2004 & & 0.16494299 \\
\hline 2005 & & 0.15158854 \\
\hline 2006 & & 0.13931532 \\
\hline 2007 & & 0.1280358 \\
\hline 2008 & & 0.1176695 \\
\hline
\end{tabular}

prepare necessary capacity of health centres and technical equipment. It involves organisation of rescue fire brigade and medical services as well take the view of administrative work.

This way it is possible to determine data from all spheres of interest. However, the prerequisite is access to information from the past. Data including machine or equipment failures as well as their causes can lead to the series of measures necessary for their elimination. Natural disasters data enable realisation of adequate building, organisational and other measures.

In case of optimum crisis management strategy determination it is mostly necessary to solve conflict between needs and possibilities. Neglect of some of the risk factors can have far-reaching consequences in case of crisis situation origin. Sensible implementation of scientific methods in crisis planning implies optimum solution of emergency situations.

This article has been supported by Agency for Science and Technology by means of financial aid No. 20-001902. and VEGA No. $1 / 0077 / 03$.

\section{References}

[1] SOUŠEK, R.: Crisis management of transport (in Czech), Institute of Jan Perner, o.p.s 2002, Pardubice, ISBN 978-80-86530-06-2.

[2] VORLÍČEK, M.: Selected chapters from mathematical statistics (in Czech), Ministerstvo národní obrany Praha, 1968.

[3] KOŽÍŠEK, J.: Mathematical statistics and statistics analysis (in Czech), Ediční středisko ČVUT Praha, 1981.

[4] ŠIMÁK, Ladislav: Crisis management in public administration (in Slovak), Žilina, FŠI ŽU, RVS, 2001. ISBN 80-88829-13-5.

[5] BRANDALÍK, F., KLUVÁNEK, P.: Operation analysis in railway transport (in Slovak), ALFA, Bratislava 